\title{
Effect of Vitamin D Levels on Bone Remodeling in Healthy Women
}

\author{
Sandhya Nair ${ }^{1}$, Hetal Bhadricha ${ }^{1}$, Sonam Hatkar ${ }^{1}$, Seema S Kadam ${ }^{1}$, Anushree Patil ${ }^{2}$, Suchitra Surve ${ }^{2}$, \\ Beena Joshi ${ }^{3}$, M. Ikram Khatkhatay ${ }^{1}$ and Meena Desai ${ }^{1, *}$ \\ ${ }^{1}$ Molecular Immunodiagnostics Division, ICMR-National Institute for Research in Reproductive Health, Mumbai, India \\ ${ }^{2}$ Department of Clincal Research, ICMR-National Institute for Research in Reproductive Health, Mumbai, India \\ ${ }^{3}$ Department of Operational Research, ICMR-National Institute for Research in Reproductive Health, Mumbai, India \\ "Corresponding author: Molecular Immunodiagnostics Division, ICMR-National Institute for Research in Reproductive Health, J. M. Street, Mumbai, India. Tel: +91-2224192141, \\ Email: mends153@gmail.com
}

Received 2019 December 31; Revised 2020 March 26; Accepted 2020 March 30.

\begin{abstract}
Background: Vitamin D deficiency is prevalent among Indian women. Subclinical vitamin D deficiency is a significant risk factor for osteopenia and fractures. However, its effect on bone metabolism and bone mineral density (BMD) is still debatable.

objectives: This study aimed to determine relationships of the vitamin D status with bone turnover markers, carboxy-terminal telopeptide of type-I collagen (CTX), N-terminal propeptide of type I procollagen (PINP), and BMD in healthy Indian women.

Methods: In this cross-sectional study, we determined serum levels of 25-hydroxy vitamin D (25(OH)D), parathyroid hormone, serum CTX, and PINP using commercial ELISA kits in 310 healthy Indian women aged 25 - 65 years who underwent BMD measurements with DXA scan.

Results: The prevalence of vitamin D deficiency was 53.87\% and vitamin D insufficiency $31.29 \%$. A direct correlation of BMD with vitamin D levels was not observed. PINP negatively correlated with vitamin D in both premenopausal (Spearman's $r=-0.169$, $\mathrm{P}<$ 0.05 ) and postmenopausal (Spearman's $\mathrm{r}=-0.241, \mathrm{P}<0.05$ ) women. However, CTX positively correlated with vitamin $\mathrm{D}$ in both premenopausal (Spearman's $r=0.228, \mathrm{P}<0.01$ ) and postmenopausal (Spearman's $\mathrm{r}=0.244, \mathrm{P}<0.05$ ) women.

Conclusions: Vitamin D deficiency is more prevalent in premenopausal women than in postmenopausal ones. Although vitamin D does not show any association with BMD, it affects bone remodeling, which is reflected by changes in the bone formation marker PINP and the bone resorption marker CTX.
\end{abstract}

Keywords: Vitamin D Deficiency, Bone Turnover Markers, Osteoporosis

\section{Background}

An adequate amount of vitamin $\mathrm{D}$ is essential for the maintenance of good bone and skeletal health as well as for calcium homeostasis (1). Vitamin D deficiency can be an important risk factor for osteomalacia and osteoporosis in adults. It is associated with secondary hyperparathyroidism and enhanced bone turnover, eventually leading to bone loss (2). It is extensively prevalent in the Indian population, and the importance of understanding the effects of vitamin D deficiency is well documented in several studies $(3,4)$.

Bone cells express vitamin D receptors, thereby denoting an important role of vitamin $\mathrm{D}$ in bone remodeling (5). The amplitude of bone remodeling can be determined using the bone formation marker N-terminal propeptide of type I procollagen (PINP) and the bone resorption marker serum carboxy-terminal telopeptide of type- I collagen (sCTX). Serum 25(OH)D concentrations are considered to be the best functional indicator of vitamin D status $(6,7)$. The relationship between serum 25(OH)D levels with bone turnover markers and bone mineral density (BMD) in women residing in different geographical areas is debatable. A recent study has shown a negative correlation of only the bone formation marker with vitamin $\mathrm{D}(8)$. In contrast, another study has shown a negative correlation of both bone resorption and formation markers with vitamin D levels, post supplementation (9). A majority of studies did not find any correlation between either bone markers and vitamin D (10-12). A study on Saudi nationals found a positive correlation of BMD with vitamin $\mathrm{D}(13)$, whereas another study on the same population observed no correlation between them (14).

\section{Objectives}

Considering the controversial results regarding the relationship between vitamin D levels and BMD in different 
populations, the present study evaluated the vitamin D status and bone health in healthy Indian women. The aim was to determine whether vitamin D insufficiency or deficiency affects bone turnover and BMD by considering the relationship between bone turnover markers and BMD in vitamin D sufficient, insufficient, and deficient subjects.

\section{Methods}

\subsection{Study Design}

This cross-sectional observational study was conducted at the Department of Molecular Immunodiagnostics of the National Institute for Research in Reproductive Health Institute, and the study protocol was approved by the Institutional Ethics Committee for Clinical Studies. Camps were organized near the study site for creating awareness about the importance of vitamin D and osteoporosis, encouraging women to participate in the study. A total of 310 healthy Indian women, aged 25 - 65 years, were recruited in the study after obtaining written informed consent from them. Women on bone compromising drugs, women with hepatic or renal disorders, metabolic bone disease, hypercortisolism, sterility, oligomenorrhea, diabetes, and malignancy, as well as pregnant and lactating women, were excluded. The clinical history of the participants and their demographic data, including age, height, weight, body mass index(BMI), menopausal status, and menopause duration, were recorded. Menopause was defined as the natural cessation of menstruation for more than 12 months. To reduce pre-analytical variations during the estimation of bone markers, a fasting blood sample was collected from all the participants between 0900 $1000 \mathrm{hrs}$, serum was separated, and aliquots were frozen at $-80^{\circ} \mathrm{C}$ for further analysis. Serum Ca, and P levels were estimated in an auto-analyzer using reagents from ERBA (Mannheim, Germany). Serum levels of 25(OH) vitamin D (Calbiotech, USA; Sensitivity: $0.67 \mathrm{ng} / \mathrm{mL}$ ), parathyroid hormone (PTH) (Biomerica Inc., USA; Sensitivity: $0.67 \mathrm{ng} / \mathrm{mL}$ ), Serum Crosslaps, CTX-I (Immunodiagnostic Systems, UK; Sensitivity: $0.020 \mathrm{ng} / \mathrm{mL}$ ), and PINP (Elabscience, USA; Sensitivity: $9.38 \mathrm{pg} / \mathrm{mL}$ ) were estimated using commercial ELISA kits. The study cohort was classified based on menopausal status as premenopausal or postmenopausal, and each group was further classified based on serum vitamin D levels as sufficient ( $\geq 30 \mathrm{ng} / \mathrm{mL}$ ), insufficient (16 - $29 \mathrm{ng} / \mathrm{mL}$ ), and deficient ( $\leq 15 \mathrm{ng} / \mathrm{mL}$ ).

\subsection{Bone Mineral Density}

All the participants underwent BMD measurement at the lumbar spine, hip, and its sub-regions with a Lunar
iDXA densitometer (GE, Healthcare) by a trained operator. Quality control procedures were carried out following the manufacturer's recommendations, and results were expressed as T-score. The instrument variation was determined regularly with a weekly calibration procedure using a phantom supplied by the manufacturer. According to the World Health Organization (WHO) criteria, BMD values were stratified as normal, osteopenic, and osteoporotic based on T-score.

\subsection{Statistical Analysis}

All statistical analyses were performed with Graph Pad Prism version 5.01. Means \pm standard deviation (SD) were used to express standard descriptive statistics. The nonparametric test used as the data did not show Gaussian distribution. Differences in the median were investigated using either the Mann-Whitney test or the Kruskal-Wallis test with Dunn's post hoc analysis. Spearman's rank correlation was calculated to indicate the strength of the relation between the two parameters. A $p$-value of less than 0.05 was considered statistically significant.

\section{Results}

\subsection{Anthropometric Data}

The basic anthropometric data of the study cohort are given in Table 1. The mean age of the women was 41.54 \pm 11.55 years, and their mean BMI was $24.75 \pm 4.78 \mathrm{~kg} / \mathrm{m}^{2}$. The mean BMD at the spine was $1.02 \pm 0.166 \mathrm{~g} / \mathrm{cm}^{2}$, and the mean BMD at the femoral neck (FN) was $0.876 \pm 0.123$ $\mathrm{g} / \mathrm{cm}^{2}$. All the women had normal levels of Ca and P, while the mean $25(\mathrm{OH}) \mathrm{D}$ level was $18.95 \pm 15.35 \mathrm{ng} / \mathrm{mL}$. Moreover, the mean PTH, CTX, and PINP levels were $59.23 \pm 23.37$ $\mathrm{pg} / \mathrm{mL}, 0.457 \pm 0.299 \mathrm{ng} / \mathrm{mL}$, and $37.35 \pm 16.24 \mathrm{ng} / \mathrm{mL}$, respectively.

\subsection{Association of Vitamin D with Various Parameters}

The study cohort was also classified based on vitamin D levels as sufficient ( $\geq 30 \mathrm{ng} / \mathrm{mL}$ ), insufficient (16 - 29 $\mathrm{ng} / \mathrm{mL}$ ), and deficient ( $\leq 15 \mathrm{ng} / \mathrm{mL}$ ). According to the WHO recommendation, the postmenopausal women were classified as normal, osteopenic, and osteoporotic based on their T-scores, whereas the premenopausal women were classified as normal and with low bone mass based on their Z-score (Table 2).

In the study cohort, $59.08 \%$ of the premenopausal women had vitamin D deficiency, of which $62.99 \%$ had low bone mass. However, the prevalence of vitamin D deficiency in the postmenopausal women was less (42.12\%) compared to the premenopausal women (Table 2 ). 


\begin{tabular}{|c|c|c|c|}
\hline Characteristics & $\begin{array}{c}\text { Cohort }(\mathrm{N}= \\
310)\end{array}$ & $\begin{array}{l}\text { Premenopausal } \\
\qquad(\mathrm{N}=\mathbf{2 1 5})\end{array}$ & $\begin{array}{c}\text { Postmenopausal } \\
\quad(\mathrm{N}=95)\end{array}$ \\
\hline Age, years & $41.54 \pm 11.55$ & $34.32 \pm 8.57$ & $52.10 \pm 5.69$ \\
\hline BMI, $\mathrm{kg} / \mathrm{m}^{2}$ & $24.75 \pm 4.78$ & $23.92 \pm 4.33$ & $25.66 \pm 4.55$ \\
\hline $\begin{array}{l}\text { BMD Spine, } \\
\mathrm{g} / \mathrm{cm}^{2}\end{array}$ & $1.02 \pm 0.166$ & $1.089 \pm 0.132$ & $0.913 \pm 0.146$ \\
\hline BMD FN, $\mathbf{g} / \mathbf{c m}^{2}$ & $0.876 \pm 0.123$ & $0.907 \pm 0.114$ & $0.808 \pm 0.116$ \\
\hline T-score spine & $-1.38 \pm 1.33$ & $-0.81 \pm 1.06$ & $-2.31 \pm 1.17$ \\
\hline T-score FN & $-1.12 \pm 0.88$ & $-0.93 \pm 0.8$ & $-1.65 \pm 0.81$ \\
\hline $\begin{array}{l}\text { Calcium, } \\
\mathrm{mg} / \mathrm{dL}\end{array}$ & $9.24 \pm 0.25$ & $9.18 \pm 1.02$ & $9.19 \pm 0.94$ \\
\hline $\begin{array}{l}\text { Phosphorous, } \\
\text { mg/dL }\end{array}$ & $5.17 \pm 1.35$ & $5.06 \pm 1.39$ & $5.08 \pm 1.12$ \\
\hline $\begin{array}{l}\text { Vitamin D, } \\
\mathrm{ng} / \mathrm{mL}\end{array}$ & $18.95 \pm 15.35$ & $18.71 \pm 17.09$ & $41.63 \pm 16.82$ \\
\hline PTH, pg/mL & $57.45 \pm 17.76$ & $55.50 \pm 13.46$ & $58.19 \pm 19.27$ \\
\hline sCTX, ng/mL & $0.457 \pm 0.299$ & $0.401 \pm 0.234$ & $0.515 \pm 0.263$ \\
\hline PINP, ng/mL & $37.35 \pm 16.24$ & $38.91 \pm 15.81$ & $59.19 \pm 19.27$ \\
\hline
\end{tabular}

Abbreviations: BMD, bone mineral density; BMI, body mass index; FN, femoral neck; PINP, N-terminal propeptide of type I procollagen; PTH, parathyroid hormone; sCTX, serum carboxy-terminal telopeptide of type-I collagen.

${ }^{\mathrm{a}}$ Values are expressed as mean $\pm \mathrm{SD}$.

We further checked whether BMD at the spine and FN, biochemical parameters serum Ca and P, PTH, and bone turnover markers PINP and sCTX varied between the sufficient, insufficient, and deficient groups of premenopausal and postmenopausal women, and the results are summarized in Tables 3 and 4.

The vitamin D status did not affect BMD at the spine or FN in both premenopausal and postmenopausal women. However, levels of $\mathrm{Ca}$ and $\mathrm{P}$ varied significantly among the three groups in the premenopausal women, as compared to the postmenopausal women. Moreover, levels of intact PTH and PINP were significantly different among the three groups in the premenopausal and postmenopausal women, but post hoc test results were non-significant. In addition, sCTX levels varied significantly between the three groups in both premenopausal and postmenopausal women.

\subsection{Correlation of Vitamin D Levels with Study Parameters}

Vitamin D levels were correlated with age, BMI, BMD, biochemical parameters, and bone turnover markers, as depicted in Table 5. Interestingly, sCTX positively correlated with vitamin D in the premenopausal (Spearman's $\mathrm{r}$ $=-0.228, \mathrm{P}<0.01$ ) and postmenopausal (Spearman's $\mathrm{r}=$ $0.244, \mathrm{P}<0.05$ ) women.

\section{Discussion}

Vitamin D deficiency is widespread in India, with its prevalence ranging from $70 \%-100 \%$ (15). The cut-off for vitamin D sufficiency in our study was considered as serum $25(\mathrm{OH})$ vitamin levels $\geq 30 \mathrm{ng} / \mathrm{mL}$ and deficiency as $\leq 15$ $\mathrm{ng} / \mathrm{mL}$. The reason is that PTH levels fall down considerably at this threshold value of vitamin $\mathrm{D}$, as indicated in many cross-sectional studies (16). Vitamin D deficiency was about 1.4 times more prevalent among the premenopausal women compared to postmenopausal women. This is consistent with other Indian studies, where women at reproductive age have a higher prevalence of vitamin $\mathrm{D}$ deficiency (17-19). This could be attributed to low dietary calcium and vitamin D intake, limited exposure to sunlight, socio-cultural factors, geographical factors, level of pollution, clothing, pigmentation of the skin, and time spent outdoors, which affect the production of subcutaneous vitamin D (20).

Vitamin D levels did not show any correlation with BMD in our study. There has been a lack of consensus on the relationship of vitamin D with BMD. Few studies have reported the association of vitamin D with BMD in the south Asian population (21-23). However, other studies have indicated a lack of association between vitamin D and BMD in the same population $(18,24,25)$. The disparity can be attributed to differences in ethnicity, age group, and differences in cut-off limits to define vitamin D deficiency and insufficiency.

Calcium levels had an inverse correlation with vitamin D, whereas serum phosphorous was positively correlated with vitamin D in premenopausal women. Serum calcium increases due to enhanced bone resorption, and demineralization carried out using PTH in the vitamin D deficiency state (26). Although the exact mechanism of phosphorous release and reclamation is not clear in various organs, the positive effect is due to phosphate regulation carried out with vitamin $\mathrm{D}, \mathrm{PTH}$, and fibroblast growth factor 23 (FGF23), collectively called calciotropic and phosphpotropic hormone, by modulating intestinal phosphate absorption and renal phosphate reabsorption (27).

A significant negative correlation was observed between vitamin D and PINP. An increase in PTH levels leads to an increase in PINP levels, indicating that bone remodeling requires an adequate amount of PTH $(8,28)$. Hence, the increase in PINP levels is due to the increase in PTH levels. Unlike other studies, which showed either negative (8) or no (29) correlation of CTX with vitamin D, we observed a positive correlation between SCTX and vitamin D with a marked effect observed in premenopausal women. This is in line with a study carried out in Tehran on healthy adolescents (30). CTX levels were high in females less than 20 
Table 2. The vitamin D status in Premenopausal and Postmenopausal Women ${ }^{\mathrm{a}, \mathrm{b}}$

\begin{tabular}{|c|c|c|c|c|c|c|}
\hline \multirow{2}{*}{ Characteristics } & \multirow{2}{*}{ Values } & \multicolumn{2}{|c|}{ Premenopausal $(\mathrm{N}=\mathbf{2 1 5})$} & \multicolumn{3}{|c|}{ Postmenopausal ( $\mathrm{N}=95)$} \\
\hline & & Normal BMD & Low BMD & Normal & Osteopenia & Osteoporosis \\
\hline Sufficient & $46(14.84)$ & $29(90.62)$ & $3(9.38)$ & $1(7.14)$ & $4(28.57)$ & $9(64.29)$ \\
\hline Insufficient & $97(31.29)$ & $53(94.64)$ & $3(5.36)$ & $2(4.87)$ & $17(41.46)$ & $22(53.65)$ \\
\hline Deficient & $167(53.87)$ & $121(95.27)$ & $6(4.73)$ & $6(15)$ & $17(42.5)$ & $17(42.5)$ \\
\hline
\end{tabular}

${ }^{\mathrm{a}}$ Values are expressed as No. (\%).

${ }^{b}$ Frequency in parenthesis: the premenopausal women classified as normal (Z-score $>-2.0$ ) and low BMD (Z-score $<-2.0$ ); the postmenopausal women classified as normal (T-score $>-1.0$ ), osteopenic (T-score -1.0 to -2.5), and osteoporotic (T-score $\leq-2.5$ ); sufficient (vitamin D $\geq 30 \mathrm{ng} / \mathrm{mL}$ ), insufficient (vitamin D $16-29 \mathrm{ng} / \mathrm{mL}$ ), and deficient (vitamin $\mathrm{D} \leq 15 \mathrm{ng} / \mathrm{mL}$ )

Table 3. BMD and Biochemical Parameters in Premenopausal Women Classified Based on Vitamin D Levels ${ }^{\mathrm{a}}$

\begin{tabular}{|c|c|c|c|c|}
\hline Parameters & Sufficient $(\mathrm{N}=32)$ & Insufficient $(\mathrm{N}=56)$ & Deficient $(N=127)$ & PValue \\
\hline Age, years & $42(36-46)$ & $34(30-41)^{\mathrm{b}}$ & $33(25-39)^{b}$ & 0.0001 \\
\hline BMD spine, $\mathrm{g} / \mathrm{cm}^{2}$ & $1.056(0.88-1.292)$ & $1.075(0.845-1.428)$ & $1.052(0.837-1.571)$ & 0.645 \\
\hline BMD FN, $\mathbf{g} / \mathrm{cm}^{2}$ & $0.876(0.685-1.113)$ & $0.923(0.687-1.206)$ & $0.886(0.648-1.291)$ & 0.678 \\
\hline Calcium, mg/dL & $8.4(7.5-10.5)$ & $8.7(6.2-11.7)$ & $9.5(7.1-11.4)^{b, c}$ & 0.001 \\
\hline Phosphorous, mg/dL & $4.65(3.6-6.72)$ & $5.26(3.4-11.44)$ & $4.61(2.5-12.84)^{d}$ & 0.003 \\
\hline PTH, pg/mL & $53.68(21.04-60.45)$ & $64.29(39.68-107.2)$ & $64.89(42.69-112.6)$ & 0.038 \\
\hline PINP, pg/mL & $27.62(6.04-67.22)$ & $36.82(13.94-83.6)$ & $36.25(12.94-95.1)$ & 0.034 \\
\hline sCTX, ng/mL & $0.444(0.069-1.093)$ & $0.433(0.153-0.945)$ & $0.326(0.016-1.157)^{\mathrm{d}, \mathrm{e}}$ & 0.002 \\
\hline
\end{tabular}

Abbreviations: BMD, bone mineral density; FN, femoral neck, PINP, N-terminal propeptide of type I procollagen; PTH, parathyroid hormone; sCTX, serum carboxyterminal telopeptide of type-I collagen.

${ }^{a}$ Values are expressed as median (range)

${ }^{\mathrm{b}} \mathrm{P}<0.001$ compared to sufficient.

${ }^{\mathrm{c}} \mathrm{P}<0.001$ compared to insufficient.

${ }^{\mathrm{d}} \mathrm{P}<0.05$ compared to insufficient.

${ }^{\mathrm{e}} \mathrm{P}<0.05$ compared to sufficient.

Table 4. BMD and Biochemical Parameters in Postmenopausal Women Classified Based on Vitamin D Levels ${ }^{\mathrm{a}}$

\begin{tabular}{|c|c|c|c|c|}
\hline Parameters & Sufficient (N=14) & Insufficient $(\mathbf{N}=\mathbf{4 1})$ & Deficient $(N=40)$ & P Value \\
\hline Age, years & $53(44-61)$ & $52(41-65)$ & $53(42-63)$ & 0.849 \\
\hline BMD spine, $\mathrm{g} / \mathrm{cm}^{2}$ & $0.889(0.640-1.099)$ & $0.890(0.576-1.286)$ & $0.911(0.672-1.296)$ & 0.868 \\
\hline BMD FN, $\mathbf{g} / \mathbf{c m}^{2}$ & $0.817(0.652-1.032)$ & $0.815(0.567-0.956)$ & $0.774(0.612-1.194)$ & 0.461 \\
\hline Calcium, mg/dL & $9.4(7.2-11.1)$ & $9.1(7.7-11.3)$ & $8.55(8-10.6)$ & 0.147 \\
\hline Phosphorous, mg/dL & $5.06(4.06-6.17)$ & $5.04(2.8-9.39)$ & $4.87(3.3-8.12)$ & 0.482 \\
\hline PTH, pg/mL & $44.43(19.04-70.99)$ & $52.41(23.01-106.8)$ & $60.01(30.98-112.1)$ & 0.032 \\
\hline PINP, pg/mL & $36.95(22.20-53.08)$ & $31.24(10.90-51.6)$ & $34.7(21.68-72.76)^{\mathrm{b}}$ & 0.028 \\
\hline sCTX, ng/mL & $0.759(0.308-0.953)$ & $0.434(0.03-0.940)^{c}$ & $0.444(0.06-1.05)$ & 0.029 \\
\hline
\end{tabular}

Abbreviations: BMD, bone mineral density; FN, femoral neck, PINP, N-terminal propeptide of type I procollagen; PTH, parathyroid hormone; sCTX, serum carboxyterminal telopeptide type-I collagen.

${ }^{\mathrm{a}}$ Values are expressed as median (range).

${ }^{\mathrm{b}} \mathrm{P}<0.05$ compared to insufficient.

${ }^{\mathrm{c}} \mathrm{P}<0.05$ compared to sufficient.

years, decreased between the age range of 20 - 49 years, and increased afterward (31). A cross-sectional study on Indian women indicated that hypovitaminosis $\mathrm{D}$ existed in about $88 \%$ of women at reproductive age (20 - 49 years) (17).
Low sCTX and vitamin D in the premenopausal women of our cohort may justify the strong positive association between them. Surprisingly, we found a higher level of vitamin D in the postmenopausal women compared to the pre- 
Table 5. The Correlation Between Vitamin D with BMD and Biochemical Parameters in the Premenopausal and Postmenopausal Women ${ }^{\mathrm{a}}$

\begin{tabular}{|c|c|c|}
\hline \multirow{2}{*}{ Parameters } & \multicolumn{2}{|c|}{ Vitamin $D, \mathbf{n g} / \mathbf{m L}$} \\
\hline & $\begin{array}{c}\text { Premenopausal }(\mathrm{N}= \\
215)\end{array}$ & $\begin{array}{c}\text { Postmenopausal }(\mathrm{N}= \\
95)\end{array}$ \\
\hline Age, years & $0.257^{\mathrm{b}}$ & 0.071 \\
\hline BMI, $\mathrm{kg} / \mathrm{m}^{2}$ & $0.143^{c}$ & 0.018 \\
\hline BMD spine, $\mathrm{g} / \mathrm{cm}^{2}$ & 0.012 & 0.009 \\
\hline BMD FN, $\mathbf{g} / \mathrm{cm}^{2}$ & 0.051 & 0.072 \\
\hline Calcium, mg/dL & $-0.256^{\mathrm{b}}$ & -0.169 \\
\hline Phosphorous, mg/dL & $0.184^{c}$ & 0.148 \\
\hline PTH, pg/mL & $-0.315^{c}$ & $-0.236^{c}$ \\
\hline PINP, pg/mL & $-0.169^{c}$ & $-0.241^{c}$ \\
\hline sCTX, ng/mL & $0.228^{\mathrm{b}}$ & $0.244^{\mathrm{c}}$ \\
\hline
\end{tabular}

Abbreviations: BMI, body mass index; BMD, bone mineral density; FN, femoral neck, PINP, N-terminal propeptide of type I procollagen; PTH, parathyroid hormone; sCTX, serum carboxy-terminal telopeptide of type-I collagen.

${ }^{a}$ Values expressed as Spearman's correlation coefficient.

${ }^{\mathrm{b}}$ P value $<0.001$

${ }^{\mathrm{c}}$ P value $<0.05$.

menopausal ones. This could be due to the intake of vitamin D supplements, which was not noted. Hence, a positive association of vitamin D with sCTX was observed.

There were some limitations to the study. Firstly, the confounding factors for bone turnover markers were not adjusted during analysis. Secondly, we could not determine whether the participants, especially the postmenopausal women, were on vitamin D supplementation, which could be the reason for the lower prevalence of vitamin D deficiency in postmenopausal women.

Nevertheless, this observational study reveals that although vitamin D does not affect BMD directly, it influences the bone remodeling process. In our study, vitamin D was observed to have a positive correlation with CTX and a negative correlation with PINP. Bone turnover markers varied across the three groups classified based on vitamin D levels based on. Thus, vitamin D levels may be a predisposing risk factor for osteoporosis and should be considered along with BMD measurements for therapeutic purposes.

\section{Footnotes}

Authors' Contribution: Study concept and design as well as revision of the manuscript: MD and MIK. Data analysis and interpretation as well as drafting of the manuscript: SN and HB. Lab investigations: SN, SH, and SK. Participant recruitment for the study: SS, AP, and BJ.

Conflict of Interests: The authors do not have any conflicts of interest.
Ethical Approval: The NIRRH Ethics Committee for clinical studies (no.: 216/2012).

Funding/Support: ICMR-NIRRH intramural funding.

Informed Consent: The participants were encouraged to participate in the study with the help of organizing camps and by briefing them about the work. Interested participants were called at the study site, given detailed information about the study, and asked whether they were willing to participate. Those who agreed to participate were given the informed consent form for signing. The informed consent form was also available in the local languages to promote the participants' understanding.

\section{References}

1. Holick MF. Vitamin D and bone health.J Nutr.1996;126(4 Suppl):1159S64S. doi:10.1093/jn/126.suppl_4.1159S. [PubMed: 8642450].

2. Chandran M, Hoeck HC, Wong HC, Zhang RF, Dimai HP. Vitamin D status and its relationship with bone mineral density and parathyroid hormone in Southeast Asian adults with low bone density. Endocr Pract. 2011;17(2):226-34. doi:10.4158/EP10202.OR. [PubMed: 21041169].

3. Harinarayan CV, Joshi SR. Vitamin D status in India-its implications and remedial measures. J Assoc Physicians India. 2009;57:40-8. [PubMed: 19753759].

4. Mithal A, Wahl DA, Bonjour JP, Burckhardt P, Dawson-Hughes B, Eisman JA, et al. Global vitamin D status and determinants of hypovitaminosis D. Osteoporos Int. 2009;20(11):1807-20. doi: 10.1007/s00198009-0954-6. [PubMed: 19543765].

5. Zarei A, Morovat A, Javaid K, Brown CP. Vitamin D receptor expression in human bone tissue and dose-dependent activation in resorbing osteoclasts. Bone Res. 2016;4:16030. doi: 10.1038/boneres.2016.30. [PubMed: 27785371]. [PubMed Central: PMC5057180].

6. Kuo TR, Chen $\mathrm{CH}$. Bone biomarker for the clinical assessment of osteoporosis: Recent developments and future perspectives. Biomark Res. 2017;5:18. doi: 10.1186/s40364-017-0097-4. [PubMed: 28529755]. [PubMed Central: PMC5436437].

7. Cashman KD, van den Heuvel EG, Schoemaker RJ, Preveraud DP, Macdonald HM, Arcot J. 25-Hydroxyvitamin D as a biomarker of vitamin D Status and its modeling to inform strategies for prevention of vitamin D deficiency within the population. Adv Nutr. 2017;8(6):94757. doi: 10.3945/an.117.015578. [PubMed: 29141976]. [PubMed Central: PMC5682995].

8. Marwaha RK, Garg MK, Mithal A, Gupta S, Shukla M, Chadha A. Effect of vitamin D supplementation on bone turnover markers in children and adolescents from North India. Indian J Endocrinol Metab. 2019;23(1):27-34. doi: 10.4103/ijem.IJEM_149_18. [PubMed: 31016149]. [PubMed Central: PMC6446672].

9. Eckard AR, O'Riordan MA, Rosebush JC, Ruff JH, Chahroudi A, Labbato D, et al. Effects of vitamin D supplementation on bone mineral density and bone markers in HIV-infected youth. J Acquir Immune Defic Syndr. 2017;76(5):539-46. doi: 10.1097/QAI.0000000000001545. [PubMed: 28902705]. [PubMed Central: PMC5680140].

10. Molgaard C, Larnkjaer A, Cashman KD, Lamberg-Allardt C, Jakobsen J, Michaelsen KF. Does vitamin D supplementation of healthy Danish Caucasian girls affect bone turnover and bone mineralization? Bone. 2010;46(2):432-9. doi: 10.1016/j.bone.2009.08.056. [PubMed: 19735754].

11. Barnes MS, Robson PJ, Bonham MP, Strain JJ, Wallace JM. Effect of vitamin D supplementation on vitamin D status and bone turnover markers in young adults. Eur J Clin Nutr. 2006;60(6):727-33. doi: 10.1038/sj.ejcn.1602374. [PubMed: 16391584]. 
12. Hill KM, Laing EM, Hausman DB, Acton A, Martin BR, McCabe GP, et al. Bone turnover is not influenced by serum 25-hydroxyvitamin D in pubertal healthy black and white children. Bone. 2012;51(4):7959. doi: 10.1016/j.bone.2012.06.014. [PubMed: 22750015]. [PubMed Central: PMC4292920].

13. Sadat-Ali M, Al Elq AH, Al-Turki HA, Al-Mulhim FA, Al-Ali AK. Influence of vitamin D levels on bone mineral density and osteoporosis. Ann Saudi Med. 2011;31(6):602-8. doi: 10.4103/0256-4947.87097. [PubMed: 22048506]. [PubMed Central: PMC3221132].

14. Alkhenizan A, Mahmoud A, Hussain A, Gabr A, Alsoghayer S, Eldali A. The rELATIonship between $25(\mathrm{OH})$ D Levels (Vitamin D) and bone mineral density (BMD) in a Saudi population in a community-based setting. PLoS One. 2017;12(1). e0169122. doi: 10.1371/journal.pone.0169122. [PubMed: 28046015]. [PubMed Central: PMC5207714]

15. G R, Gupta A. Vitamin D deficiency in India: Prevalence, causalities and interventions. Nutrients. 2014;6(2):729-75. doi: 10.3390/nu6020729. [PubMed: 24566435]. [PubMed Central: PMC3942730]

16. Chapuy MC, Preziosi P, Maamer M, Arnaud S, Galan P, Hercberg S, et al. Prevalence of vitamin D insufficiency in an adult normal population. Osteoporos Int. 1997;7(5):439-43. doi: 10.1007/s001980050030. [PubMed: 9425501].

17. Sofi NY, Jain M, Kapil U, Seenu V, Ramakrishnan L, Yadav CP, et al. Status of serum vitamin $\mathrm{D}$ and calcium levels in women of reproductive age in national capital territory of India. Indian J Endocrinol Metab. 2017;21(5):731-3. doi: 10.4103/ijem.IJEM_134_17. [PubMed: 28989883]. [PubMed Central: PMC5628545].

18. Harinarayan CV, Sachan A, Reddy PA, Satish KM, Prasad UV, Srivani P. Vitamin D status and bone mineral density in women of reproductive and postmenopausal age groups: A cross-sectional study from South India. J Assoc Physicians India. 2011;59:698-704. [PubMed: 22616335].

19. Sachan A, Gupta R, Das V, Agarwal A, Awasthi PK, Bhatia V. High prevalence of vitamin D deficiency among pregnant women and their newborns in northern India. Am J Clin Nutr. 2005;81(5):1060-4. doi: 10.1093/ajcn/81.5.1060. [PubMed: 15883429].

20. Webb AR. Who, what, where and when-influences on cutaneous vitamin D synthesis. Prog Biophys Mol Biol. 2006;92(1):17-25. doi: 10.1016/j.pbiomolbio.2006.02.004. [PubMed: 16766240].

21. Cranney A, Horsley T, O’Donnell S, Weiler H, Puil L, Ooi D, et al. Effectiveness and safety of vitamin D in relation to bone health. Evid Rep Technol Assess (Full Rep). 2007;(158):1-235. [PubMed: 18088161]. [PubMed Central: PMC4781354].

22. Arya V, Bhambri R, Godbole MM, Mithal A. Vitamin D status and its relationship with bone mineral density in healthy Asian Indians. Osteo- poros Int. 2004;15(1):56-61. doi: 10.1007/s00198-003-1491-3. [PubMed: 13680103].

23. Roy DK, Berry JL, Pye SR, Adams JE, Swarbrick CM, King Y, et al. Vitamin D status and bone mass in UK South Asian women. Bone. 2007;40(1):200-4. doi: 10.1016/j.bone.2006.07.004. [PubMed: 16950669].

24. Hamson C, Goh L, Sheldon P, Samanta A. Comparative study of bone mineral density, calcium, and vitamin D status in the Gujarati and white populations of Leicester. Postgrad Med J. 2003;79(931):279-83. doi: 10.1136/pmj.79.931.279. [PubMed: 12782775]. [PubMed Central: PMC1742698].

25. Marwaha RK, Tandon N, Reddy DH, Mani K, Puri S, Aggarwal N, et al. Peripheral bone mineral density and its predictors in healthy school girls from two different socioeconomic groups in Delhi. Osteoporos Int. 2007;18(3):375-83. doi: 10.1007/s00198-006-0247-2. [PubMed: 17160694].

26. Thiering E, Bruske I, Kratzsch J, Hofbauer LC, Berdel D, von Berg A, et al Associations between serum 25-hydroxyvitamin D and bone turnover markers in a population based sample of German children. Sci Rep. 2015;5:18138. doi: 10.1038/srep18138. [PubMed: 26667774]. [PubMed Central: PMC4678865].

27. Lee JJ, Plain A, Beggs MR, Dimke H, Alexander RT. Effects of phosphoand calciotropic hormones on electrolyte transport in the proximal tubule. F1000Res. 2017;6:1797. doi:10.12688/f1000research.12097.1. [PubMed: 29043081]. [PubMed Central: PMC5627579].

28. Bauer DC, Garnero P, Bilezikian JP, Greenspan SL, Ensrud KE, Rosen CJ et al. Short-term changes in bone turnover markers and bone mineral density response to parathyroid hormone in postmenopausal women with osteoporosis. J Clin Endocrinol Metab. 2006;91(4):1370-5. doi: 10.1210/jc.2005-1712. [PubMed: 16449339].

29. Schwetz V, Trummer C, Pandis M, Grubler MR, Verheyen N, Gaksch $M$, et al. Effects of vitamin D supplementation on bone turnover markers: A randomized controlled trial. Nutrients. 2017;9(5). doi: 10.3390/nu9050432. [PubMed: 28448457]. [PubMed Central: PMC5452162].

30. Larijani B, Hossein-Nezhad A, Feizabad E, Maghbooli Z, Adibi H, Ramezani M, et al. Vitamin D deficiency, bone turnover markers and causative factors among adolescents: A cross-sectional study. J Diabetes Metab Disord. 2016;15:46. doi: 10.1186/s40200-016-0266-2. [PubMed: 27752482]. [PubMed Central: PMC5062858].

31. Jenkins N, Black M, Paul E, Pasco JA, Kotowicz MA, Schneider HG. Age-related reference intervals for bone turnover markers from an Australian reference population. Bone. 2013;55(2):271-6. doi: 10.1016/j.bone.2013.04.003. [PubMed: 23603243]. 